\title{
MJFAS MALAYSIAN JUURNAL OF

\section{MHD free convective flow past an impulsively started vertical plate through a porous medium with radiative heat transfer and chemical reaction in a rotating system}

\author{
Uday Singh Rajput, Mohammad Shareef ${ }^{\star}$ \\ Department of Mathematics and Astronomy, University of Lucknow, Lucknow-226007, India \\ Corresponding author: rajputshareeflu@gmail.com
}

\section{Article history}

Received 24 April 2017

Revised 21 May 2018

Accepted 20 February 2019

Published Online 14 April 2019

\begin{abstract}
The effect of Hall current, radiation and chemical reaction on MHD free convective flow past an impulsively started vertical plate through a porous medium in a rotating system is considered. The analytical solutions of dimensionless governing equations are obtained by Laplace transform technique. The effects of various parameters on the fluid velocity, concentration and temperature distribution near the plate are analyzed and shown graphically. The numerical values of shear stress at the plate are shown in a table. It is observed that the fluid velocity near the plate is affected significantly by Hall current, radiation, chemical reaction and porous medium. The conclusion of the study have great applications in the field of science and engineering.
\end{abstract}

Keywords: Magnetohydrodynamics (MHD), chemical reaction, rotation, hall current

\section{INTRODUCTION}

The application of MHD viscous incompressible fluid flow through porous medium involving radiative heat transfer under the influence of chemical reaction among various species have great importance in many areas of science and engineering. Stewartson [1, 2] has studied the related problems to understand the behavior of the fluids in an unsteady boundary layer. His study was completely based on the boundary layer equations. The influence of magnetic field on such a flow within a porous and non-porous medium with radiative heat transfer has a great significance in the designing of heat exchangers, MHD pumps, MHD generator, nuclear reactors, oil exploration, space vehicle propulsion etc. In recent years due to the importance of the study, considerable progress has been made to the study of thermophysical properties affecting MHD boundary layer flow. The MHD flow of a uniformly stretched vertical permeable surface in the presence of heat generation/absorption and chemical reaction was studied by Chamka [3]. He solved the problem analytically and observed that the Prandlt number, Schmidt number and the strength of magnetic field retard the fluid velocity. Also, the radiation and mass transfer effects on two-dimensional flow past an impulsively started infinite vertical plate was studied by Prasad et al. [4]. The governing equations used by Prasad et al. [4] were solved using a finite-difference method. They [4] observed that when the radiation parameter increases, the velocity and temperature decrease in the boundary layer. Ibrahim and Makinde [5] has studied the radiation effect on chemically reacting MHD boundary layer flow of heat and mass transfer through a porous vertical flat plate and solved the problem numerically using shooting technique with the fourth order Runge-Kutta integration scheme. Makinde and Mhone [6] analyzed the effects of a transverse magnetic field and radiative heat transfer to unsteady flow of a conducting optically thin fluid through a channel filled with saturated porous medium and non-uniform wall temperature. They [6] solved the problem analytically and used to compute the rate of heat transfer and shear stress at the wall. Further, Reddy [7] worked on heat and mass transfer effects on the unsteady MHD radiative flow of a chemically reacting fluid past an impulsively started vertical plate. He [7] observed that the presence of chemical reaction retards the fluid velocity, decreases the concentration and increases the thermal boundary layer thickness.

However, if the strength of applied magnetic field is very strong, the effect of Hall current is also significant. Also, the rotating flow of viscous, incompressible and electrically conducting fluid has attracted the attention of researchers due to their abundant geophysical and astrophysical applications. It is well known that a number of astronomical bodies possess fluid interiors and magnetic fields. Therefore many scholars have studied such models, for instance, Mazumdar et al. [8] worked on flow with heat transfer in the hydrodynamic Ekman layer on a porous plate with Hall effects. Further, Agarwal et al. [9] analyzed the combined influence of dissipation and Hall effect on free convective flow in a rotating fluid. It was observed by [9] that the primary and secondary shear-stresses increases and decreases, respectively, with the increase in magnetic field and Hall parameters. Also, the work on oscillating vertical plate embedded in a porous medium with radiative heat and mass transfer is done by Kishore et al. [10].

Influenced by the above-discussed literature and applications, the model under consideration analyzes the MHD free convective flow past an impulsively started vertical plate through a porous medium with radiative heat transfer and chemical reaction in a rotating system. The problem is solved analytically using the Laplace transform technique. The relevant set of graphical results illustrating the effects of various parameters involved in the problem is presented and discussed. The numerical values of skin-friction have been tabulated. 


\section{MATHEMATICAL FORMULATION}

Consider an unsteady flow of a viscous, incompressible, electrically conducting fluid past an impulsively started vertical infinite non-conducting flat plate in a porous medium. Further we assume that a homogeneous chemical reaction of first order between the diffusing species and the fluid take place at a constant rate $K_{o}$. Let $x^{\prime}-$ axis be chosen vertically upward along the motion of plate and the $z^{\prime}$ - axis normal to the plate. The fluid and the plate rotate as a rigid body with a constant angular velocity $\Omega^{\prime}$ about $z^{\prime}$-axis. A uniform magnetic field $B_{o}$ is applied at an angle $\delta$ from the plate as shown in the Fig. 1. The fluid is assumed to be electrically conducting whose magnetic Reynolds number is very small, so the induced magnetic field produced by fluid motion is negligible in comparison to the applied one; hence $\overrightarrow{\mathbf{B}}=\left(0,0, B_{o}\right)$. As there is applied and polarization voltage are assumed to be absent, therefore electric field $\overrightarrow{\mathbf{E}}=(0,0,0)$. As the plate is of infinite extent, so all the physical variables depends only on $z^{\prime}$ and $t^{\prime}$.

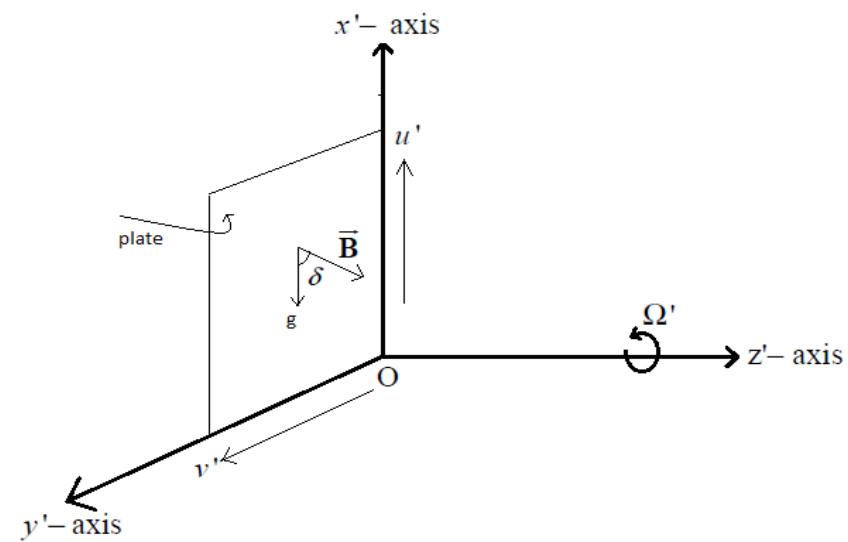

Fig. 1 Geometry of the model

Initially, at the time $t^{\prime} \leq 0$, the fluid and the plate are at rest and at a uniform temperature and concentration $T_{o}$ and $C_{o}$ respectively. At the time $t^{\prime}>0$, the plate starts moving with a constant velocity $u_{o}$ in vertically upward direction and the temperature and concentration of the plate are raised to $T_{p}$ and $C_{p}$ respectively. Here the impulsive movement of the plate and free convection causes the fluid motion. From the equation of conservation of electric charge $\vec{\nabla} \cdot \overrightarrow{\mathbf{J}}=0$, we have $J_{z^{\prime}}=$ constant, where $\overrightarrow{\mathbf{J}}=\left(J_{x^{\prime}}, J_{y^{\prime}}, J_{z^{\prime}}\right)$ is the current density vector. Since the plate is assumed to be nonconducting therefore at the plate $J_{z^{\prime}}=0$. Thus, everywhere in the fluid $J_{z^{\prime}}=0$. Here the continuity equation identically becomes zero. So, under the above assumptions, the governing equations with Boussinesq's approximation and radiative heat transfer are as follows:

$$
\begin{aligned}
& \frac{\partial u^{\prime}}{\partial t^{\prime}}-2 \Omega^{\prime} v^{\prime}=v \frac{\partial^{2} u^{\prime}}{\partial z^{\prime 2}}+g \beta\left(T-T_{0}\right)+g \beta^{*}\left(C-C_{0}\right) \\
&+\frac{B_{o} \operatorname{Sin} \delta}{\rho} J_{y^{\prime}}-\frac{v}{K^{\prime}} u^{\prime}, \\
& \frac{\partial v^{\prime}}{\partial t^{\prime}}+2 \Omega^{\prime} u^{\prime}=v \frac{\partial^{2} v^{\prime}}{\partial z^{\prime 2}}-\frac{B_{o} \operatorname{Sin} \delta}{\rho} J_{x^{\prime}}-\frac{v}{K^{\prime}} v^{\prime}, \\
& \frac{\partial T}{\partial t^{\prime}}=\frac{k}{\rho c_{p}} \frac{\partial^{2} T}{\partial z^{\prime 2}}-\frac{1}{\rho c_{p}} \frac{\partial q^{(\mathrm{r})}}{\partial z^{\prime}}, \\
& \frac{\partial C}{\partial t^{\prime}}=D \frac{\partial^{2} C}{\partial z^{\prime 2}}-K_{o}\left(C-C_{o}\right) .
\end{aligned}
$$

The boundary conditions taken are as under:

$$
\begin{aligned}
& t^{\prime} \leq 0\left\{\begin{array}{l}
u^{\prime}=0, v^{\prime}=0, T=T_{o}, C=C_{o} \forall z^{\prime} . \\
u^{\prime}=u_{o}, v^{\prime}=0, T=T_{o}+\left(T_{p}-T_{o}\right) \frac{u_{o}^{2}}{v} t^{\prime}, \\
t^{\prime}>0 \\
C=C_{o}+\left(C_{p}-C_{o}\right) \frac{u_{o}^{2}}{v} t^{\prime} \text { at } z^{\prime}=0 \text { and } \\
u^{\prime} \rightarrow 0, v^{\prime} \rightarrow 0, T \rightarrow T_{o}, C \rightarrow C_{o} \text { as } z^{\prime} \rightarrow \infty .
\end{array}\right\}
\end{aligned}
$$

Taking Hall current into account and neglecting the electron pressure gradient, the ion slip and the thermo-electric effects, the generalized Ohm's law can be written as

$$
\overrightarrow{\mathbf{J}}+\frac{\omega_{e} \tau_{e}}{B_{o}}(\overrightarrow{\mathbf{J}} \times \overrightarrow{\mathbf{B}})=\sigma(\overrightarrow{\mathbf{E}}+\overrightarrow{\mathbf{q}} \times \overrightarrow{\mathbf{B}})
$$

Where $\overrightarrow{\mathbf{q}}=\left(u^{\prime}, v^{\prime}, 0\right)$.On solving (6), we get

$$
J_{x^{\prime}}=\frac{\sigma B_{o} \operatorname{Sin} \delta\left(v^{\prime}+m u^{\prime}\right)}{1+m^{2}}, J_{y^{\prime}}=\frac{\sigma B_{o} \operatorname{Sin} \delta\left(m v^{\prime}-u^{\prime}\right)}{1+m^{2}}
$$

By using Rosseland approximation (Brewster [11]), the radiative heat flux $q^{(\mathrm{r})}$ is given by

$$
q^{(\mathrm{r})}=-\frac{4 \sigma_{s}}{3 k_{e}} \frac{\partial T^{\prime 4}}{\partial z^{\prime}}
$$

Here the symbols used are: $T$-temperature of the fluid, $C$-concentration of the fluid, $B_{o}$-external magnetic field, $\delta-$ Angle of inclination of $\overrightarrow{\mathbf{B}}$ with $x^{\prime}$-axis, $u^{\prime}$-primary velocity of the fluid, $v^{\prime}$-secondary velocity of the fluid, $K^{\prime}$ - permeability parameter, $\quad \beta$-volumetric coefficient of thermal expansion, $\beta^{*}-$ volumetric coefficient of concentration expansion, $D$-mass diffusion coefficient, $\quad q^{(\mathrm{r})}$ - radiative heat flux, $\alpha$-thermal diffusivity, $k_{e}$ - mean absorption coefficient, $\omega_{e}$-cyclotron frequency of electron, $\tau_{e}$-electron collision time, $g$-acceleration due to gravity, $\rho$-density of fluid, $v$-kinematic viscosity, $\sigma_{s}-$ Stefan-Boltzmann constant, $m\left(=\omega_{e} \tau_{e}\right)-$ Hall parameter, $J_{x^{\prime}}-$ current density along $x^{\prime}-$ axis and $J_{y^{\prime}}-$ current density along $y^{\prime}-$ axis.

If temperature difference within the flow is sufficiently small, then expanding $T^{\prime 4}$ by using Taylor series about $T_{o}$ (neglecting higher order terms), we get,

$$
T^{4} \cong 4 T_{o}^{3} T-3 T_{o}^{4}
$$

By using (7) and (8), (3) reduces to

$\frac{\partial T}{\partial t^{\prime}}=\alpha \frac{\partial^{2} T}{\partial z^{\prime 2}}+\frac{16 \sigma_{s} \alpha T_{o}^{3}}{3 k_{e} k} \frac{\partial^{2} T}{\partial z^{\prime 2}}$

To obtain the equations in dimensionless form, the following nondimensional quantities are introduced: 


$$
\begin{aligned}
& u=\frac{u^{\prime}}{u_{o}}, v=\frac{v^{\prime}}{u_{o}}, t=\frac{u_{o}^{2}}{v} t^{\prime}, z=\frac{u_{o}}{v} z^{\prime}, \theta=\frac{\left(T-T_{o}\right)}{\left(T_{p}-T_{o}\right)}, \\
& \phi=\frac{\left(C-C_{o}\right)}{\left(C_{p}-C_{o}\right)}, \mathrm{P}_{r}=\frac{v}{\alpha}, N=\frac{k_{e} k}{4 \sigma_{s} T_{o}^{3}}, K=\frac{u_{o}^{2}}{v^{2}} K^{\prime}, \\
& M^{2}=\frac{\sigma B_{o}^{2} \operatorname{Sin}^{2} \delta v}{\rho u_{o}^{2}}, G_{r}=\frac{g \beta v\left(T_{p}-T_{\infty}\right)}{u_{o}^{3}}, S_{c}=\frac{v}{D}, \\
& G_{m}=\frac{g \beta^{*} v\left(C_{p}-C_{\infty}\right)}{u_{o}^{3}}, \Omega=\frac{v}{u_{o}^{2}} \Omega^{\prime}, \mathrm{c}_{r}=\frac{K_{o}}{u_{o}^{2}} v .
\end{aligned}
$$

Here $u$ is dimensionless primary velocity of the fluid, $v$-dimensionless secondary velocity of the fluid, $z$-dimensionless spatial coordinate normal to the plate, $\phi$-dimensionless concentration of the fluid, $\theta$-dimensionless temperature of the fluid, $P_{r}$ - Prandlt number, $S_{c}$ - Scthimth number $G_{r}$ - Thermal Grashof number, $G_{m}$-Mass Grashof number, $t$-dimensionless time, $N$-Radiation parameter, $\Omega$-dimensionless rotation parameter and $M$ - magnetic field parameter.

Using equation (10), equations (1), (2), (4), (9) and (5) respectively, become:

$$
\left.\begin{array}{l}
\frac{\partial u}{\partial t}-2 \Omega v=\frac{\partial^{2} u}{\partial z^{2}}+\frac{M^{2}}{\left(1+m^{2}\right)}(m v-u)+G_{m} \phi+G_{r} \theta-\frac{u}{K}, \\
\frac{\partial v}{\partial t}+2 \Omega u=\frac{\partial^{2} v}{\partial z^{2}}-\frac{M^{2}}{\left(1+m^{2}\right)}(v+m u)-\frac{v}{K}, \\
\frac{\partial \phi}{\partial t}=\frac{1}{S_{c}} \frac{\partial^{2} \phi}{\partial z^{2}}-c_{r} \phi, \\
\frac{\partial \theta}{\partial t}=\frac{1}{R_{a} P_{r}} \frac{\partial^{2} \theta}{\partial z^{2}} . \\
t \leq 0: u=0, v=0, \theta=0, \phi=0 \quad \forall z, \\
t>0: u=1, v=0, \theta=t, \phi=t \text { at } z=0 \text { and } \\
u \rightarrow 0, v \rightarrow 0, \theta \rightarrow 0, \phi \rightarrow 0 \text { as } z \rightarrow \infty .
\end{array}\right\}
$$

To solve above system, assume $\mathbf{V}=u+i v$. Then using equations (11) and (12), we get,

$$
\frac{\partial \mathbf{V}}{\partial t}=\frac{\partial^{2} \mathbf{V}}{\partial z^{2}}-b \mathbf{V}+G_{r} \theta+G_{m} \phi
$$

The boundary conditions (15) are transformed:

$$
\begin{aligned}
& t \leq 0: \mathbf{V}=0, \theta=0, \phi=0 \quad \forall z, \\
& t>0: \mathbf{V}=1, \theta=t, \phi=t \text { at } z=0 \\
& \mathbf{V} \rightarrow 0, \theta \rightarrow 0, \phi \rightarrow 0 \quad \text { as } z \rightarrow \infty \text {. }
\end{aligned}
$$

The governing non-dimensional partial differential equations (16), (14) and (13) subject to the above boundary conditions prescribed in equation (17) are solved using the Laplace Transform technique.

The solution of the model is as below:

$$
\begin{aligned}
\mathbf{V}(z, t) & =\psi_{1}(\mathrm{t})\left\{\operatorname{Cosh}\left(F_{8} z\right)+\xi_{1}(z, \mathrm{t}) e^{-F_{8} z}-\xi_{2}(z, \mathrm{t}) e^{F_{8} z}\right\} \\
& +F_{3} e^{-B_{1} t}\left\{-\operatorname{Cosh}\left(F_{2} z\right)+\xi_{3}(z, \mathrm{t}) e^{-F_{2} z}-\xi_{4}(z, \mathrm{t}) e^{F_{2} z}\right\} \\
+ & \left(2 F_{4} z+H_{7} z\right)\left\{-\operatorname{Sinh}\left(F_{8} z\right)+\xi_{1}(z, \mathrm{t}) e^{-F_{8} z}+\xi_{2}(z, \mathrm{t}) e^{F_{8} z}\right\} \\
& -F_{5} z \sqrt{t} e^{-\frac{F_{1}^{2} z^{2}}{4 t}}-\xi_{7}(z, \mathrm{t}) \operatorname{Erfc}\left(\frac{F_{1} z}{2 \sqrt{t}}\right)
\end{aligned}
$$

$$
\begin{gathered}
+F_{3} e^{-B_{1} t}\left\{\operatorname{Cosh}\left(F_{6} z\right)+\xi_{5}(z, \mathrm{t}) e^{-F_{6} z}-\xi_{6}(z, \mathrm{t}) e^{F_{6} z}\right\} \\
-H_{9} e^{-B_{2} t}\left\{\operatorname{Cosh}\left(H_{2} z\right)-\xi_{8}(z, \mathrm{t}) e^{-H_{2} z}-\xi_{9}(z, \mathrm{t}) e^{H_{2} z}\right\} \\
-H_{8} z\left\{-\operatorname{Sinh}\left(H_{1} z\right)+\xi_{10}(z, \mathrm{t}) e^{-H_{1} z}+\xi_{11}(z, \mathrm{t}) e^{H_{1} z}\right\} \\
+\psi_{2}(\mathrm{t})\left\{-\operatorname{Cosh}\left(H_{1} z\right)-\xi_{10}(z, \mathrm{t}) e^{-H_{1} z}+\xi_{11}(z, \mathrm{t}) e^{H_{1} z}\right\} \\
+H_{9} e^{-B_{2} t}\left\{\operatorname{Cosh}\left(H_{3} z\right)+\xi_{12}(z, \mathrm{t}) e^{-H_{3} z}-\xi_{13}(z, \mathrm{t}) e^{H_{3} z}\right\} \\
\theta(z, t)=-\frac{e^{-\frac{z^{2} R_{a}}{4 t}} z \sqrt{t R_{a}}}{\sqrt{\pi}}+\frac{1}{2}\left(2 t+z^{2} R_{a}\right) \operatorname{Erfc}\left(\frac{z \sqrt{R_{a}}}{2 t}\right), \\
\phi(z, t)=\frac{e^{-z \sqrt{c_{r} S_{c}}}}{4 \sqrt{c_{r}}}\left[\left(2 t \sqrt{c_{r}}-z \sqrt{S_{c}}\right)\left\{1+\operatorname{Erf}\left(\sqrt{c_{r} t}-\frac{z}{2} \sqrt{\frac{S_{c}}{t}}\right)\right\}\right. \\
\left.+e^{2 z \sqrt{c_{r} S_{c}}}\left(2 t \sqrt{c_{r}}+z \sqrt{S_{c}}\right) \operatorname{Erfc}\left(\sqrt{c_{r} t}+\frac{z}{2} \sqrt{\frac{S_{c}}{t}}\right)\right] .
\end{gathered}
$$

The dimensionless skin-friction components $\tau_{x}$ and $\tau_{y}$ are obtained as:

$$
\tau_{x}+i \tau_{y}=-\left.\frac{\partial \mathbf{V}}{\partial z}\right|_{z=0}
$$

\section{RESULTS AND DISCUSSION}

In order to explain the significance of the study a representative set of numerical results is shown graphically in Fig. 2 to 17. It is noticed from Fig. 2 to 11 that magnitudes of primary velocity $u$ and secondary velocity $v$ attain distinctive maximum values near the surface of the plate and then start decreasing. Fig. 4 and 5 show the effect of buoyancy force on the velocity where we observe that both the components of the velocity get increased by thermal Grashof number and mass Grashof number. From Fig. 6 and 7, it is observed that on increasing the permeability parameter $K$ of the porous medium, both the components of velocity increase. It validates that an increase in $K$ implies a decrease in the resistance of the porous medium resulting in accelerating primary and secondaey velocities. Effect of Hall current on flow behavior can be seen from Fig. 8 and 9. Here we see that on increasing the Hall current parameter $m$, the primary velocity increases rapidly near the plate whereas secondary velocity decreases throughout the boundary layer region. This shows that Hall current tends to accelerate primary velocity in the region near the surface of the plate whereas it tends to retard secondary velocity throughout the boundary layer region. Fig. 10 and 11 show the effect of radiation parameter $N$ on both components of velocity, here it is observed that it retards the flow. Also, the temperature of the fluid decreases with radiation parameter (Fig. 12). Hence we conclude that an increase in the value of radiation parameter, both the momentum and thermal boundary layer thicknesses decrease. The effect of chemical reaction parameter $c_{r}$ on the primary velocity, secondary velocity and concentration are shown in Fig. 2, 3 and 15 respectively. From the Fig. 1 and 15, we observe that both the components of velocity and concentration decrease with $c_{r}>0$. In Fig. 16, it can be seen that the concentration of the fluid is inversely proportional to the value of Schmidt number $S_{c}$. Thus, the increase in $S_{c}$ reduces the concentration in the system. A similar effect can be seen for temperature profile with Prandlt number $P_{r}$ (Fig. 13). Also concentration and temperature boundary layer increase with time (Fig. 14 and 17).

The effects of various parameters on the skin-friction are shown in Table-1. It is found from table-1, that the value of $\tau_{x}$ increases when the values of $M, N, \delta$ and $c_{r}$ are increased (keeping other parameters fixed) but if the value of $m$ is increased, it gets decreased. Also, it is 
observed that magnitude of $\tau_{y}$ increases with $M$ and $\delta$. And it

decreases when $m, c_{r}$ and $N$ are increased.

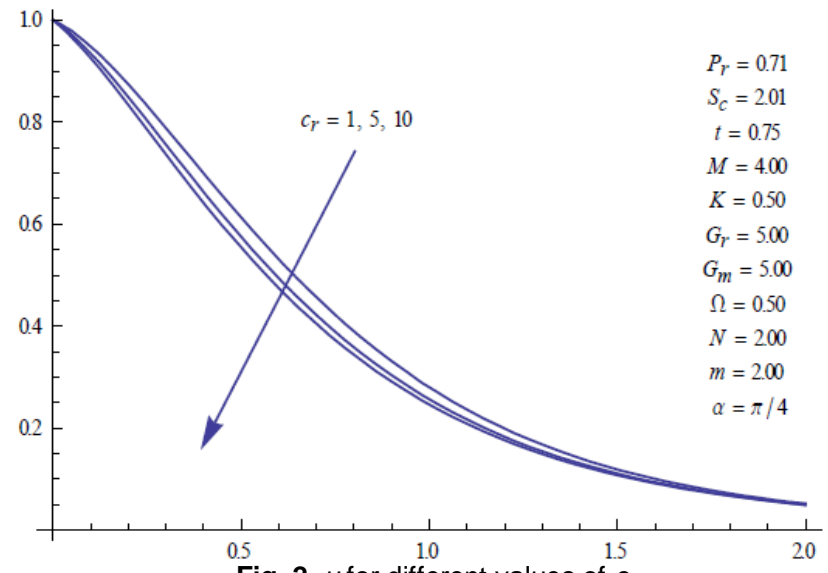

Fig. $2 u$ for different values of $c_{r}$.

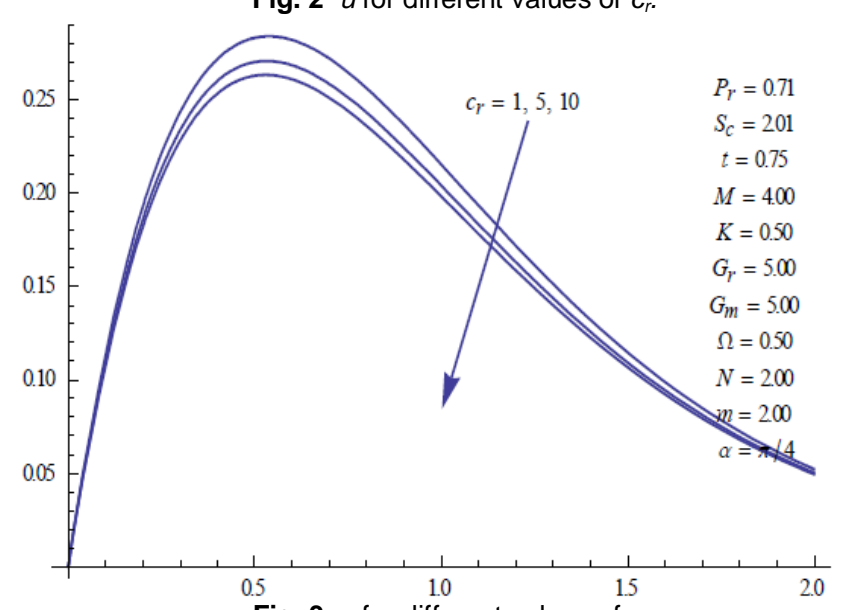

Fig. $3 v$ for different values of $c_{r}$.

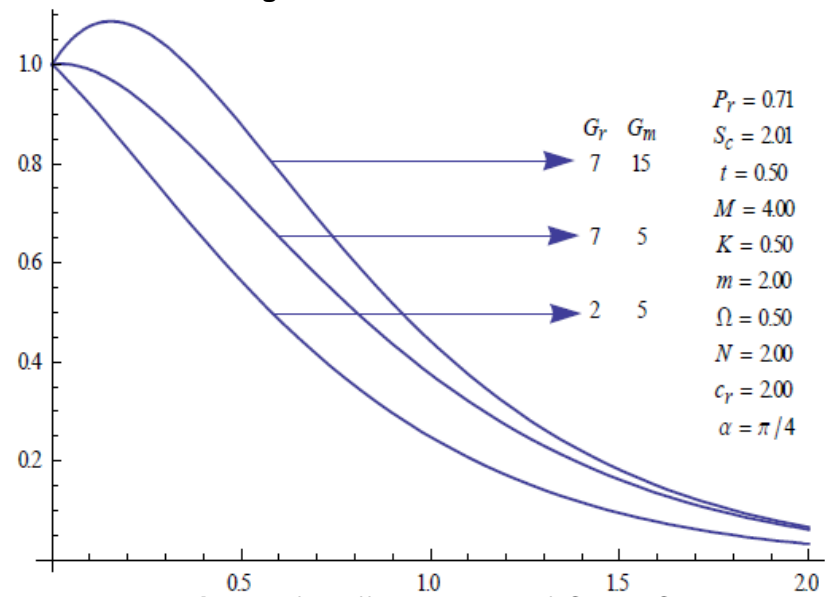

Fig. $4 u$ for different values of $G_{r}$ and $G_{m}$.

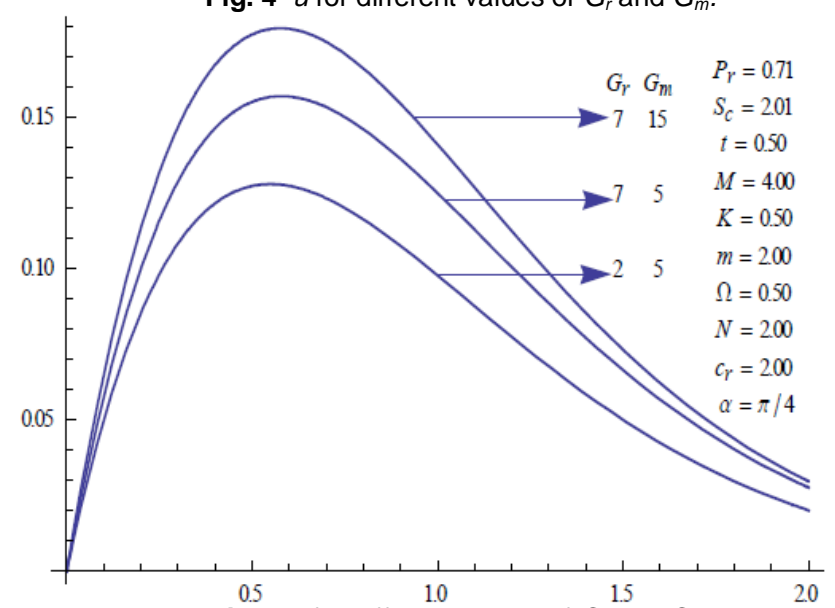

Fig.5 $v$ for different values of $G_{r}$ and $G_{m}$.
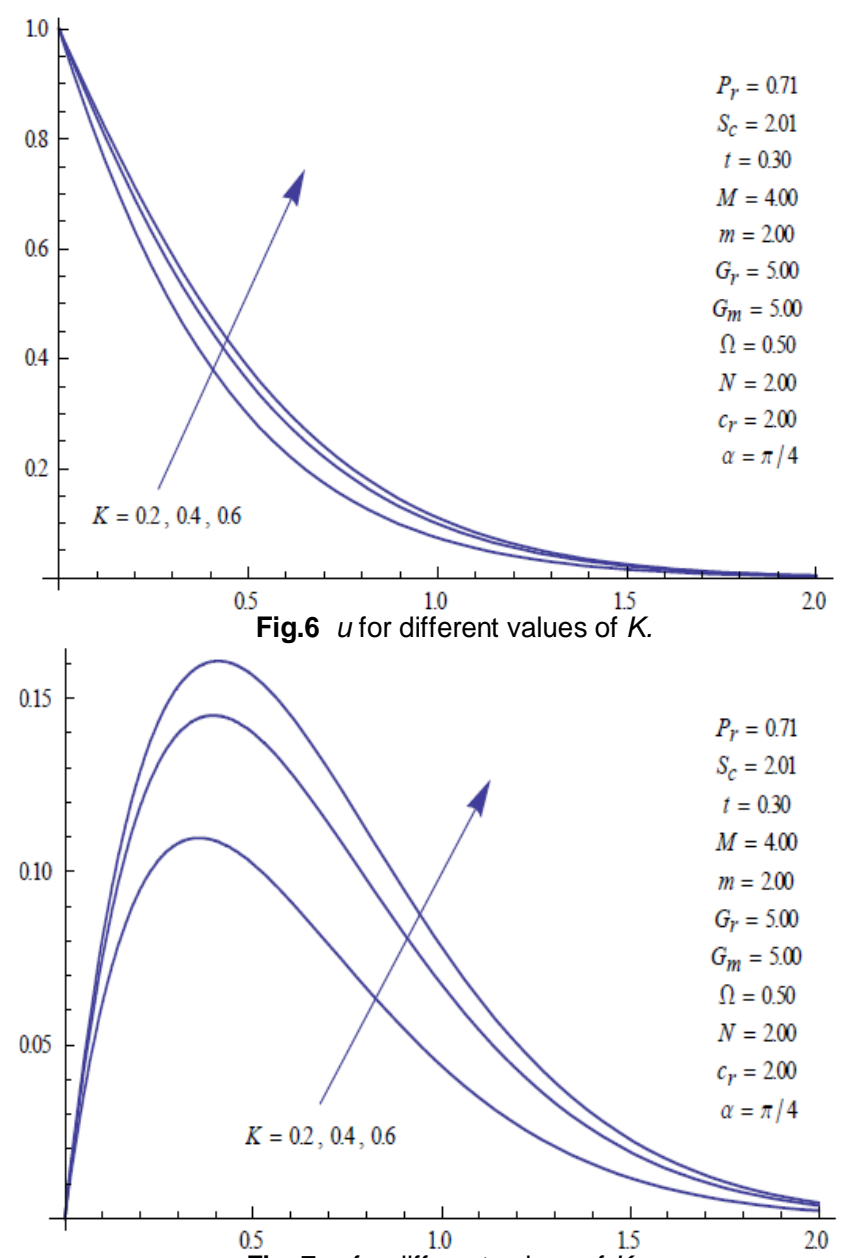

Fig. $7 v$ for different values of $K$.

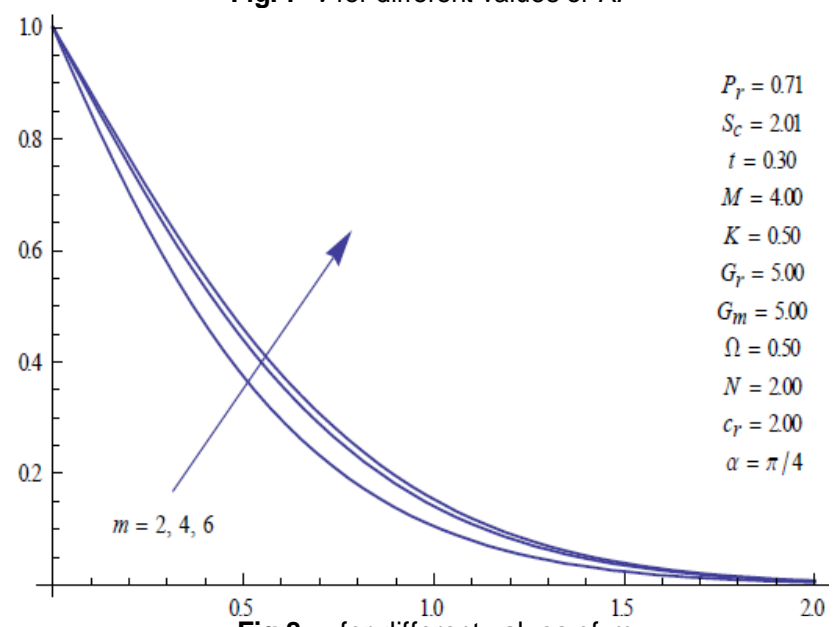

Fig.8 $u$ for different values of $m$.

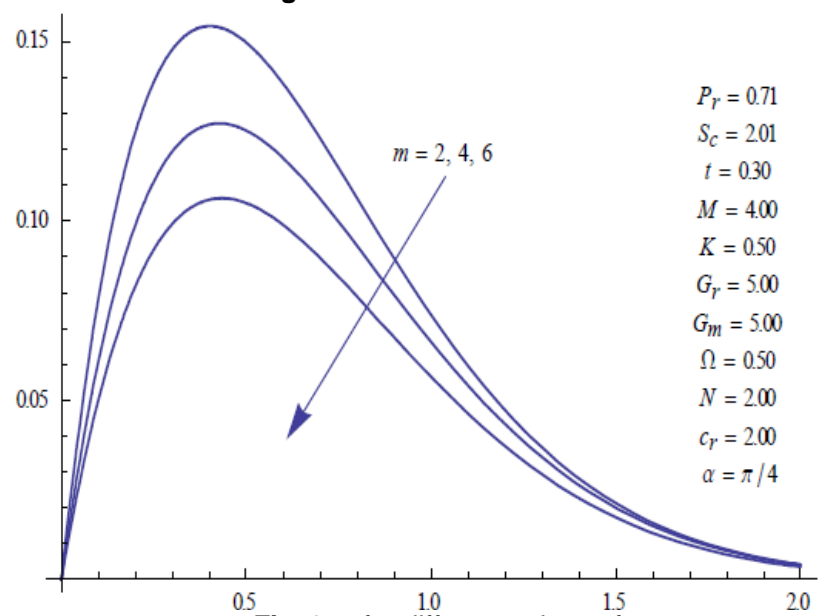

Fig. $9 v$ for different values of $m$. 


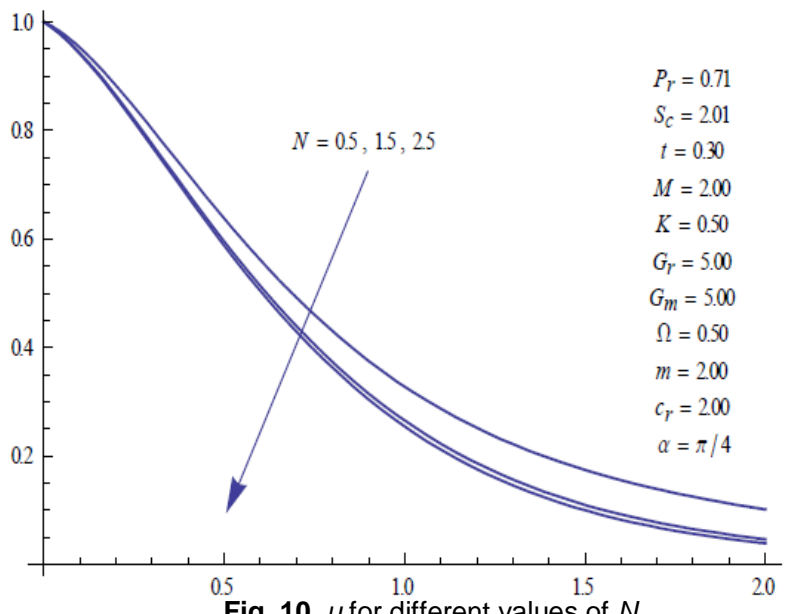

Fig. $10 u$ for different values of $N$
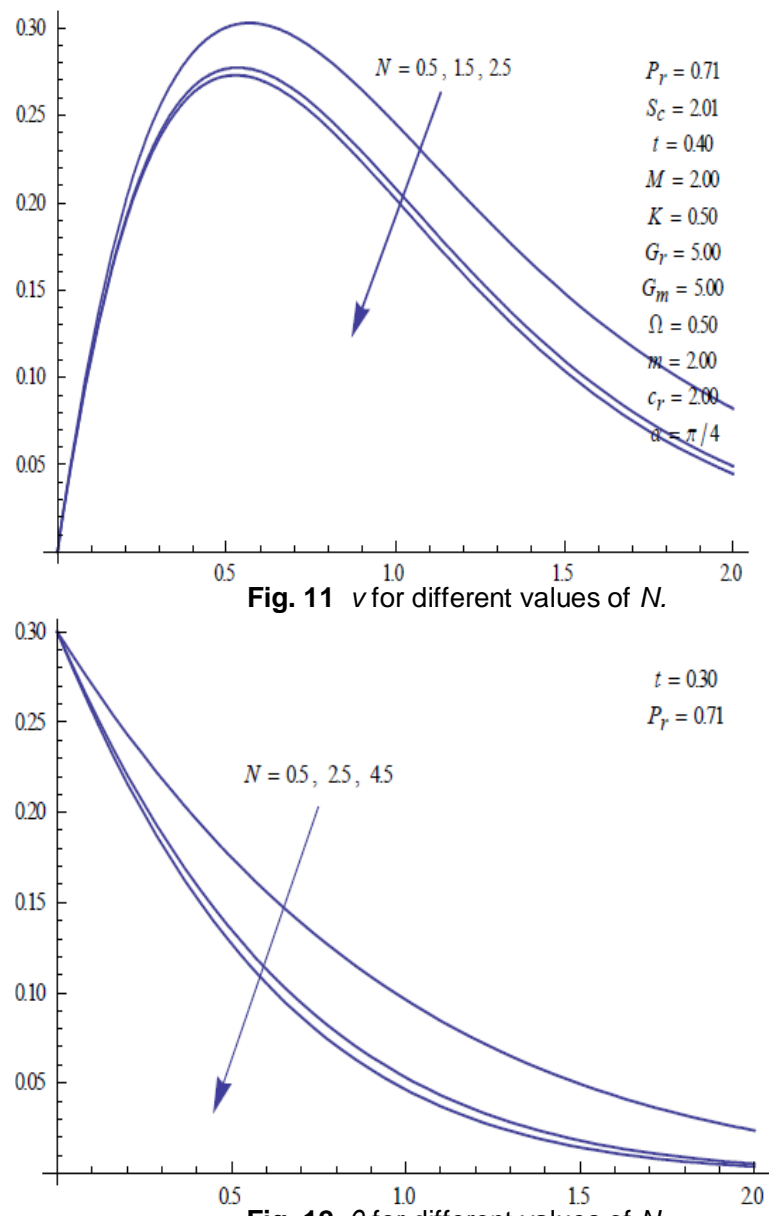

Fig. $12 \theta$ for different values of $N$.

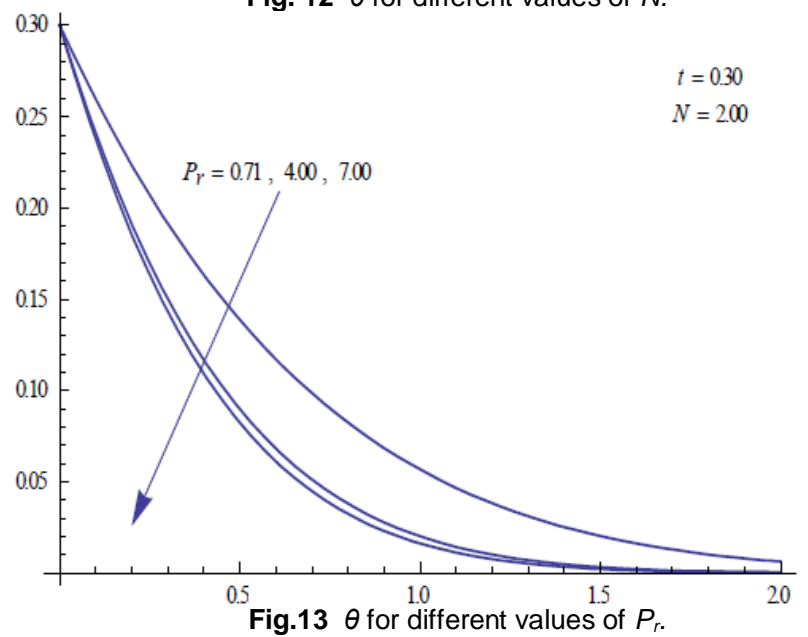

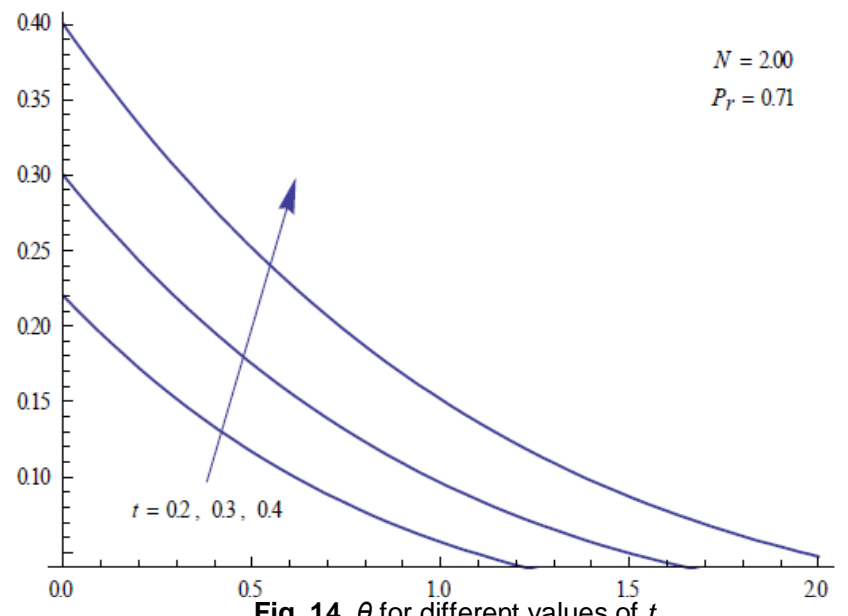

Fig. $14 \theta$ for different values of $t$.
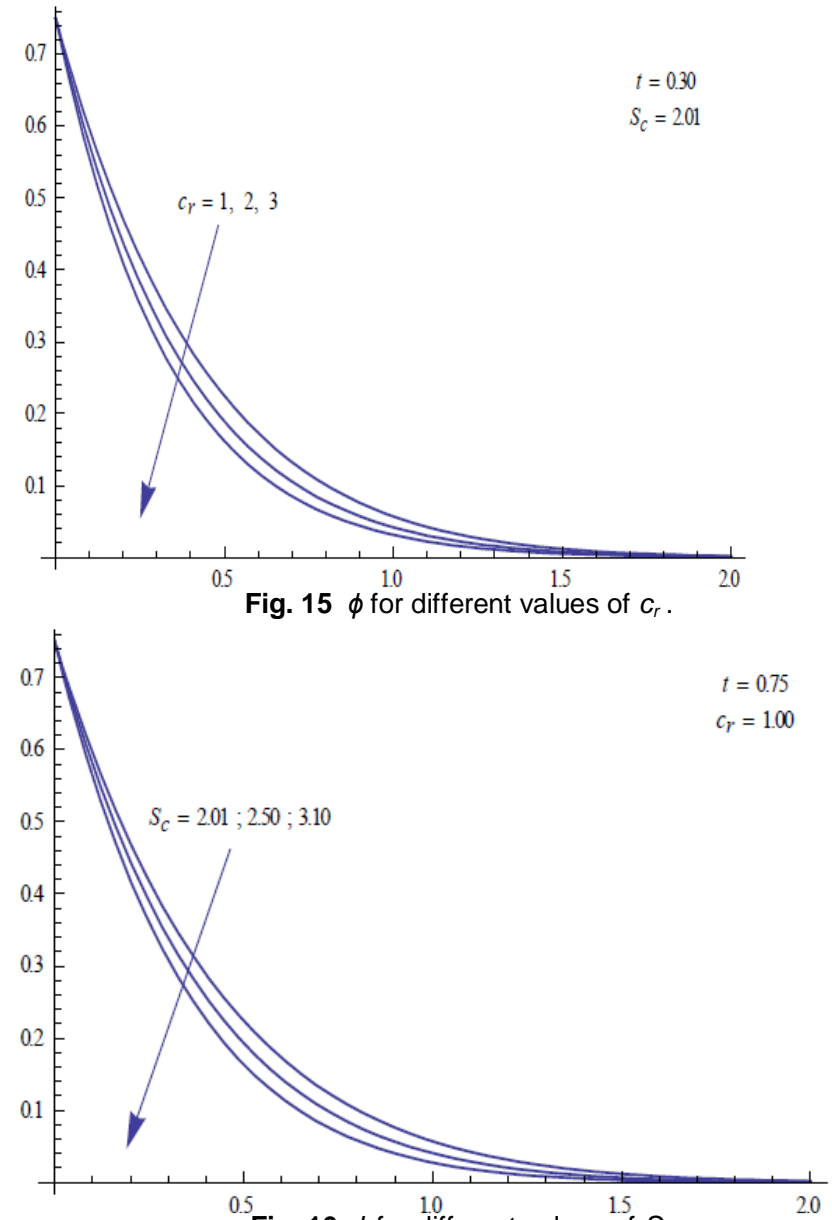

${ }^{0.5}$ Fig. $16 \phi$ for different values of $S_{c}$.

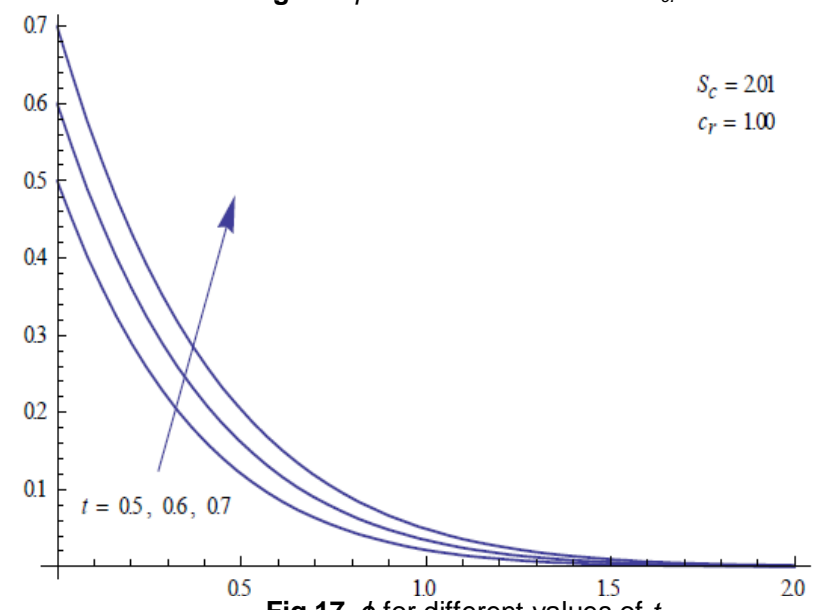

Fig.17 $\phi$ for different values of $t$ 
Table 1 Skin friction for different parameters.

\begin{tabular}{|c|c|c|c|c|c|c|}
\multicolumn{6}{c|}{$\left(P_{r}=0.71, \mathrm{~S}_{c}=2.01, t=0.2, \mathrm{~K}=0.5, G_{r}=5, \mathrm{G}_{m}=5, \Omega=0.5\right)$} \\
\hline$m$ & $M$ & $C_{r}$ & $N$ & $\delta$ & $\tau_{x}$ & $-\tau_{y}$ \\
\hline 1.5 & 2 & 2 & 0.5 & $45^{\circ}$ & 1.5473 & 0.4248 \\
\hline 2.5 & 2 & 2 & 0.5 & $45^{\circ}$ & 1.4666 & 0.3811 \\
\hline 2 & 4 & 2 & 0.5 & $45^{\circ}$ & 1.8355 & 0.8660 \\
\hline 2 & 6 & 2 & 0.5 & $45^{\circ}$ & 2.4250 & 1.4674 \\
\hline 2 & 2 & 1 & 0.5 & $45^{\circ}$ & 1.4940 & 0.4030 \\
\hline 2 & 2 & 3 & 0.5 & $45^{\circ}$ & 1.4995 & 0.4027 \\
\hline 2 & 2 & 2 & 1 & $45^{\circ}$ & 1.5116 & 0.4015 \\
\hline 2 & 2 & 2 & 2 & $45^{\circ}$ & 1.5233 & 0.4005 \\
\hline 2 & 2 & 2 & 0.5 & $30^{\circ}$ & 1.4436 & 0.3175 \\
\hline 2 & 2 & 2 & 0.5 & $60^{\circ}$ & 1.5512 & 0.4858 \\
\hline
\end{tabular}

\section{CONCLUSION}

It is found that Hall current has a tendency to accelerate the primary velocity whereas it retards the secondary velocity. When the radiation parameter increases, the velocity and temperature decrease in the boundary layer. The skin friction increases with the increase in radiation parameter. The effects of chemical reaction and radiation parameter are similar on the flow. Further it is seen that the flow in both the directions is accelerated by increasing the porosity of the medium. It is as per the expectation of the flow.

\section{APPENDIX}

$b=\frac{M^{2}(\operatorname{Sin} \delta)^{2} i}{m+i}+\frac{1}{K}+2 i \Omega, R_{a}=\frac{3 N}{3 N+4}, A_{1}=\frac{G_{r}}{1-P_{r} R_{a}}$,

$A_{2}=\frac{G_{m}}{1-S_{c}}, B_{1}=\frac{b}{1-P_{r} R_{a}}, B_{2}=\frac{b-S_{c} c_{r}}{1-S_{c}}, \mathrm{~F}_{1}=\sqrt{P_{r} R_{a}}$,

$\psi_{1}(\mathrm{t})=1+\frac{A_{1}}{B_{1}^{2}}+\frac{A_{2}}{B_{2}^{2}}-\frac{A_{1} t}{B_{1}}-\frac{A_{2} t}{B_{2}}, \psi_{2}(\mathrm{t})=\frac{A_{2}}{B_{2}^{2}}-\frac{A_{2} t}{B_{2}}$,

$F_{2}=\sqrt{b-\mathrm{B}_{1}}, \mathrm{~F}_{3}=\frac{A_{1}}{B_{1}^{2}}, F_{4}=\frac{A_{1}}{4 \sqrt{b} B_{1}}, F_{5}=\frac{A_{1}}{B_{1}} \sqrt{\frac{R_{a} P_{r}}{\pi}}$,

$$
\begin{aligned}
& F_{6}=\sqrt{-B_{1} P_{r} R_{a}}, F_{7}=\sqrt{-B_{1}}, F_{8}=\sqrt{b}, H_{1}=\sqrt{c_{r} S_{c}}, \\
& \mathrm{H}_{2}=\sqrt{b-B_{2}}, \mathrm{H}_{3}=\sqrt{S_{c}\left(-B_{2}+c_{r}\right)} .
\end{aligned}
$$

\section{REFERENCES}

[1] Stewartson, K. 1951. On the impulsive motion of a flat plate in a viscous fluid. Part 1. The Quarterly Journal of Mechanics \&Applied Mathematics, 4(2), 182-198.

[2] Stewartson, K. 1973. On the impulsive motion of a flat plate in a viscous fluid. Part 2. The Quarterly Journal of Mechanics \&Applied Mathematics, 22(2), 143-152.

[3] Chamka, A. J. 2003. MHD flow of a uniformly stretched vertical permeable surface in the presence of heat generation/absorption and chemical reaction. International Communication in Heat Mass Transfer, 30(3), 413-422.

[4] Prasad, V. R., Bhaskar Reddy, N. and Muthucumaraswamy, R. 2007. Radiation and mass transfer effects on two-dimensional flow past an impulsively started infinite vertical plate. International Journal of Thermal Sciences 46(2007) 1251-1258.

[5] Ibrahim, S. Y.and Makinde, O.D. 2011. Radiation effect on chemically reacting (MHD) boundary layer flow of heat and mass transfer through a porous vertical flat plate. International Journal of the Physical Sciences, 6(6), $1508-1516$.

[6] Makinde, O. D. and Mhone, P.Y. 2005. Heat transfer to MHD oscillatory flow in a channel filled with porous medium. Romania Journal of Physics 50(9-10). 931-938.

[7] Reddy M. G. 2014. Heat and mass transfer effects on unsteady MHD radiative flow f a chemically reacting fluid past an impulsively started vertical plate. Matematika, 30(1), 1-15.

[8] Mzumdar, B. S., Gupta, A. S. and Dattaa, N. 1976. Flow and heat transfer in hydrodynamic ekman layer on a porous plate with Hall effects. Inernational Journal of Heat and Mass Transfer, 19(5), 523.

[9] Agarwal, H. L., Ram, P. C. and Singh, V. 1983. Combined influence of dissipation and Hall effect on free convective in a rotating fluid. Indian Journal of Pure and Applied Mathematics, 14(3), 314-32.

[10] Kishore, P. M., Rajesh, V. and Verma, V. S. 2012. The effects of thermal radiation and viscous dissipation on MHD heat and mass diffusion flow past an oscillating vertical plate embedded in a porous medium with variable surface conditions. Theoretical and Applied Mechanics, 39(2), 99-125.

[11] Brewster, M. Q. 1992. Thermal Radiative Transfer and Properties. New York, John Wiley \& Sons. 